\title{
WRONSKIANS AND SUBSPACES OF CERTAIN FOURTH ORDER DIFFERENTIAL EQUATIONS
}

\author{
G.J. ETGEN \\ Department of Mathematics \\ University of Houston \\ Houston, Texas 77004 U.S.A. \\ W.E. TAYLOR, JR. \\ Department of Genera1 Academics \\ Texas A \& M University at Galveston \\ Mitche11 Campus, P.0. Box 1675 \\ Galveston, Texas 77553 \\ (Received August 21, 1978)
}

ABSTRACT. The objectives of the paper are to study the behavior of Wronskians of solutions of the fourth order differential equations and to relate this behavior with the oscillations of these equations, as well as to the structure of the subspaces of the solution spaces of the equations.

KEY WORDS AND PHRASES. Ordinary Differential Equations, Wronskian, Oscillatory or non-oscillatory Solutions.

1980 MATHEMATICS SUBJECT CLASSIFICATION CODES. $34 \mathrm{C} 10$. 


\section{INTRODUCTION.}

This paper is concerned with the fourth order differential equation

$$
\left(p(x) y^{\prime \prime}\right) "-q(x) y^{\prime \prime}-r(x) y=0,
$$

and its adjoint

$$
\left[p(x) y^{\prime \prime}-q(x) y\right]^{\prime \prime}-r(x) y=0,
$$

where $\mathrm{p}, \mathrm{q}$ and $\mathrm{r}$ are assumed to be continuous real-valued functions on the interval $\mathrm{R}^{+}=[0, \infty)$. In addition, it is assumed throughout that $\mathrm{p}>0, \mathrm{q} \geq 0$ and $\mathrm{r} \geq 0$ on $\mathrm{R}^{+}$, with $\mathrm{r}$ not identically zero on any subinterval. It is clear that if $\mathrm{q}$ is a constant, then (L) is selfadjoint; otherwise (L) is non-selfadjoint.

The objectives of the paper are to study the behavior of Wronskians of solutions of $(L)$ and $\left(L^{*}\right)$, and to relate this behavior with the oscillation of $(L)$ and $\left(L^{*}\right)$, as well as to the structure of the subspaces of the solution spaces of (L) and ( $\left.L^{*}\right)$. A nontrivial solution $y$ of $(L)\left\{\left(L^{*}\right)\right\}$ is oscillatory if the set of zeros of $y$ is not bounded above. If the set of zeros of $y$ is bounded above, implying that $y$ has only finitely many zeros on $\mathrm{R}^{+}$, then $\mathrm{y}$ is nonoscillatory. Equation (L) $\left\{\left(\mathrm{L}^{*}\right)\right\}$ is oscillatory if it has at least one nontrivial oscillatory solution. For convenience hereafter, the term "solution" shall be interpreted to mean "nontrivial solution."

Various special cases of (L) have been studied in detail. In particular, we refer to the fundamental work of W. Leighton and $Z$. Nehari [10,Part I] on the selfadjoint equation

$$
\left(p(x) y^{\prime \prime}\right) "-r(x) y=0
$$

M. Keener [7,Part I] continued the investigation of (1.1), concentrating on the oscillatory behavior of solutions. S. Ahmad [1] considered the selfadjoint equation

$$
y^{(4)}-r(x) y=0,
$$


and gave a necessary and sufficient condition for the existence of a linearly independent pair of oscillatory solutions. In [12] and [13] V. Pudei investigated the behavior of solutions of the equation

$$
y^{(4)}-q(x) y^{\prime \prime}-r(x) y=0
$$

Finally, we refer to the authors' work in [5] where sufficient conditions for the oscillation of $(L)$ and $\left(L^{*}\right)$ are given, and where the behavior of both oscillatory and nonoscillatory solutions is studied.

\section{PRELIMINARY RESULTS.}

As a notational convenience in treating the solutions of equations ( $L)$ and $\left(L^{*}\right)$, we introduce the following differential operators.

$$
\begin{gathered}
D_{0} y(x)=D_{0}^{*} y(x)=y(x), D_{1} y(x)=D_{1}^{*} y(x)=y^{\prime}(x), \\
D_{2} y(x)=p(x) y^{\prime \prime}(x), D_{3} y(x)=\left[p(x) y^{\prime \prime}(x)\right]^{\prime}, D_{4} y(x)=\left[p(x) y^{\prime \prime}(x)\right]^{\prime \prime} \\
D_{2}^{*} y(x)=p(x) y^{\prime \prime}(x)-q(x) y(x), D_{3}^{*} y(x)=\left[p(x) y^{\prime \prime}(x)-q(x) y(x)\right]^{\prime} \\
D_{4}^{*} y(x)=\left[p(x) y^{\prime \prime}(x)-q(x) y(x)\right]^{\prime \prime}
\end{gathered}
$$

Our first result is essential in the work which follows. Corresponding results for equations (1.1), (1.2), and (1.3), are given in [7], [1] and [13], respectively. The proof is a straightforward modification of the proofs of Lemmas 2.1 and 2.2 in [10].

THEOREM 2.1. If $\mathrm{y}$ is a solution of (L) $\left\{\left(\mathrm{L}^{*}\right)\right\}$ such that $\mathrm{D}_{1} \mathrm{y}(\mathrm{a}) \geq 0$ $\left\{D_{i}^{*} y(a) \geq 0\right\}, i=0,1,2,3$, for some $a \in R^{+}$, with strict inequality for at least one $i$, then $D_{i} y>0\left\{D_{i}^{*} y>0\right\}, i=0,1,2,3, D_{4} y \geq 0\left\{D_{4}^{*} y \geq 0\right\}$ on $(a, \infty)$ and

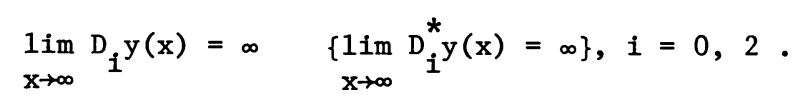

If $z$ is a solution of $(L)\left\{\left(L^{*}\right)\right\}$ such that $(-1)^{i} D_{i} z(b) \geq 0\left\{(-1)^{i} D_{i}^{*} z(b)>0\right\}$, 
$i=0,1,2,3$, for some $b \in R^{+}, b>0$, with strict inequality for at least one $i$, then $(-1)^{i} D_{i} z>0\left\{D_{i}^{*} z>0\right\}, i=0,1,2,3$, and $D_{4} z \geq 0\left\{D_{4}^{*} z \geq 0\right\}$ on $[0, b)$.

It is clear from Theorem 2.1 that $(L)$ and $\left(L^{*}\right)$ each have unbounded nonoscillatory solutions. In fact for any point $a \in R^{+}$, the four solutions $Y_{i}(x, a)$, $1=0,1,2,3$, of $(L)\left\{\left(L^{*}\right)\right\}$ determined by the initial conditions

$$
D_{i} Y_{i}(a, a)=\delta_{i j} \quad\left\{D_{j}^{*} Y_{i}(a, a)=\delta_{i j}\right\}
$$

$i, j=0,1,2,3$, where $\delta_{i j}$ is the Kronecker delta, are monotone increasing (on $[a, \infty)$ ), unbounded solutions which form a solution basis for $(L)\left\{\left(L^{*}\right)\right\}$; a so-called canonical basis. In general, a nonoscillatory solution y of $(L)\left\{\left(L^{*}\right)\right\}$ satisfying $\left.D_{1} y(x)>0, D_{1}^{*} y(x)>0\right\}, i=0,1,2,3$, on $(b, \infty)$, for some $b \in R^{+}$, is strongly increasing.

The next theorem provides the existence of a bounded nonoscillatory solution. The technique employed in establishing this result is well-known (see [7, Theorem 1.1], or [1, Theorem 2]) and, consequent1y, the proof is omitted.

THEOREM 2.2. There exists a solution $w$ of $(L)\left\{\left(L^{*}\right)\right\}$ such that

$$
\begin{aligned}
& \text { (i) } \prod_{i=0}^{3} D_{1} w(x) \neq 0 \quad\left\{\prod_{i=0}^{3} D_{i}^{*} w(x) \neq 0\right\} \text { on } R^{+} \\
& \text {(ii) } \operatorname{sgn} D_{0} w=\operatorname{sgn} D_{2} w \neq \operatorname{sgn} D_{1} w=\operatorname{sgn} D_{3} w \\
& \left\{\operatorname{sgn} D_{0^{*}}^{*}=\operatorname{sgn} D_{2}^{*} w \neq \operatorname{sgn} D_{1}^{*} w=\operatorname{sgn} D_{3}^{*} w\right\} \\
& \text { on } R^{+} \\
& \text {(iii) } \left.\lim _{x \rightarrow \infty} D_{1} w(x)=0 \quad \underset{x \rightarrow \infty}{\{1 i m} D_{i}^{*} w(x)=0\right\}, 1=0,1,2,3, \\
& \lim |w(x)|=k \geq 0
\end{aligned}
$$

A solution w of (L) $\left\{\left(L^{*}\right)\right\}$ which satisfies $(-1)^{1} D_{1} w(x)>0\left\{(-1)^{1} D_{1}^{*} w(x)>0\right\}$. $1=0,1,2,3$, on $\mathrm{R}^{+}$is said to be strongly decreasing. of course, by Theorem 2.2, each of $(L)$ and $\left(L^{*}\right)$ has such a solution. 
Pudei [13, Theorem 55] has shown that equation (1.3) is oscillatory if and only if its adjoint is oscillatory. With obvious modifications, his proof can be extended to the case of (L) and $\left(L^{*}\right)$. An alternative proof of this fact can be accomplished by showing that the $n \underline{t h}$ conjugate point of a with respect to (L) coincides with the $n \frac{t h}{n}$ conjugate point of a with respect to ( $\left.L^{*}\right)$, and then applying Leighton and Nehari's result [10, Theorem 3.8]. The final theorem in this section gives a necessary and sufficient condition for the existence of oscillatory solutions of $(L)$ and $\left(L^{*}\right)$. The authors established this result for (L) (see [5, Theorem 4.1]) using the approach developed by Ahmad in [1]. This approach can also be used to establish the result for ( $\left.L^{*}\right)$.

THEOREM 2.3. The following two statements are equivalent:

(a) Equation (L) $\left\{\left(\mathrm{L}^{*}\right)\right\}$ is oscillatory

(b) If $\mathrm{y}$ is a nonoscillatory, eventually positive solution of (L) $\left\{\left(L^{*}\right)\right\}$, then $\mathrm{y}$ is either strongly increasing or strongly decreasing.

3. WRONSKIANS OF SOLUTIONS.

Let $S$ and $S *$ denote the space of solutions of $(L)$ and ( $\left.L^{*}\right)$ respectively. The theorems of the previous section suggest the identification of the following subsets of $S$, and of $S *$ :

$$
\begin{aligned}
& I=\{y \in S \mid \text { either } y \text { or }-\mathrm{y} \text { is strongly increasing }\} \\
& D=\{w \in S \mid \text { either } w \text { or }-\mathrm{w} \text { is strongly decreasing }\} \\
& O=\{z \in S \mid z \notin S \cup D\}
\end{aligned}
$$

Let $I *, D^{*}$ and $O *$ be the corresponding subsets of $S *$. Theorems $2.1,2.2$ and 2.3 specify that none of these subsets is empty. Moreover, according to Theorem 2.3, (L) $\left\{\left(L^{*}\right)\right\}$ is oscillatory if and only if every solution in $O(O *)$ is oscillatory. In this section we study the Wronskians of solutions of (L) and (L*), we give some basic identities satisfied by these Wronskians, and we give a necessary and sufficient condition for the nonoscillation of (L) and ( $L^{*}$ ) in terms of 
these Wronskians.

Let $u, v, y, z \in S$. Then

$$
\begin{aligned}
& \mathrm{W}_{2}(\mathrm{u}, \mathrm{v})=\left|\begin{array}{ll}
\mathrm{u} & \mathrm{v} \\
\mathrm{D}_{1} \mathrm{u} & \mathrm{D}_{1} \mathrm{v}
\end{array}\right|, \mathrm{W}_{3}(\mathrm{u}, \mathrm{v}, \mathrm{y})=\left|\begin{array}{lll}
\mathrm{u} & \mathrm{v} & \mathrm{y} \\
D_{1} \mathrm{u} & D_{1} \mathrm{v} & D_{1} \mathrm{y} \\
D_{2} \mathrm{u} & D_{2} \mathrm{v} & D_{2} \mathrm{y}
\end{array}\right| \\
& \mathrm{W}_{4}(\mathrm{u}, \mathrm{v}, \mathrm{y}, \mathrm{z})=\left|\begin{array}{llll}
\mathrm{u} & \mathrm{v} & \mathrm{y} & \mathrm{z} \\
\mathrm{D}_{1} \mathrm{u} & \mathrm{D}_{1} \mathrm{v} & \mathrm{D}_{1} \mathrm{y} & \mathrm{D}_{1} \mathrm{z} \\
\mathrm{D}_{2} \mathrm{u} & \mathrm{D}_{2} \mathrm{v} & \mathrm{D}_{2} \mathrm{y} & \mathrm{D}_{2} \mathrm{z} \\
\mathrm{D}_{3} \mathrm{u} & \mathrm{D}_{3} \mathrm{v} & \mathrm{D}_{3} \mathrm{y} & \mathrm{D}_{3} \mathrm{z}
\end{array}\right|
\end{aligned}
$$

The Wronskians $w_{2}^{*}(u, v), w_{3}^{*}(u, v, y)$ and $w_{4}^{*}(u, v, y, z)$ of solutions $u, v, y, z$ of $\left(\mathrm{L}^{*}\right)$ are defined in a similar manner. Of course $\mathrm{W}_{4}(\mathrm{u}, \mathrm{v}, \mathrm{y}, \mathrm{z}) \equiv \mathrm{k}$ (constant) $\left\{\mathrm{W}_{4}^{*}(\mathrm{u}, \mathrm{v}, \mathrm{y}, \mathrm{z}) \equiv \mathrm{k}\right\}$ on $\mathrm{R}^{+}$, and $\mathrm{k} \neq 0$ if and only if $\{\mathrm{u}, \mathrm{v}, \mathrm{y}, \mathrm{z}\}$ is a solution basis for $(L)\left\{\left(L^{*}\right)\right\}$. Also, it is well-known (and easy to verify by direct calculation) that if $u, v, y$ are any three linearly independent solutions of (L) $\left\{\left(L^{*}\right)\right\}$, then $\mathrm{W}_{3}(\mathrm{u}, \mathrm{v}, \mathrm{y})\left\{\mathrm{W}_{3}^{*}(\mathrm{u}, \mathrm{v}, \mathrm{y})\right\}$ is a solution of $\left(\mathrm{L}^{*}\right)\{(\mathrm{L})\}$. The linear operators $L$ and $L^{*}$ associated with equations $(L)$ and $\left(L^{*}\right)$, respectively, are defined by

$$
\begin{aligned}
& L[y]=\left[p(x) y^{\prime \prime}\right]^{\prime \prime}-q(x) y "-r(x) y, \\
& L^{*}[y]=\left[p(x) y^{\prime \prime}-q(x) y\right]^{\prime \prime}-r(x) y
\end{aligned}
$$

A function $\mathrm{y}$ defined on $\mathrm{R}^{+}$is said to be admissable for $L\left\{L^{*}\right\}$ if each of $\mathrm{y}$ and py" $\left\{\right.$ py" - qy\} is twice differentiable on $\mathrm{R}^{+}$. If $\mathrm{y}$ is admissable for $L$ and $z$ is admissable for $L^{*}$, then they satisfy the Lagrange identity.

$$
z L[y]-y L^{*}[z]=\{y ; z\}^{\prime},
$$


where

$$
\begin{aligned}
\{y ; z\}=z\left(p y^{\prime \prime}\right) & -z^{\prime}\left(p y^{\prime \prime}\right)+\left(p z^{\prime \prime}-q z\right) y^{\prime}-\left(p z^{\prime \prime}-q z\right)^{\prime} y \\
& =\sum_{i=0}^{3}(-1)^{i} D_{i}^{*} z D_{3-i} y
\end{aligned}
$$

If follows from (3.1) that if $y \in S$ and $z \in S^{*}$, then $\{y ; z\}^{\prime}=0$, which implies $\{y ; z\}=k$ (constant) on $\mathrm{R}^{+}$. Thus the relation (3.2) determines a function $\{.,\}:. S \times S^{*} \rightarrow \mathrm{R}$ (the reals), and it is easy to verify that this function is linear in each of its arguments. This function can also be used to express a relationship between certain Wronskians of orders 3 and 4 . In particular, let $u, v, y \in S$ be linearly independent, and let $z \in S$. Then $\mathrm{w}_{3}(\mathrm{u}, \mathrm{v}, \mathrm{y}) \in \mathrm{S}^{*}$ and

$$
\left\{z ; w_{3}(u, v, y)\right\}=w_{4}(u, v, y, z)
$$

Similarly, if $\mathrm{v}, \mathrm{y}, \mathrm{z} \in S^{*}$ are linearly independent and $\mathrm{u} \epsilon S^{*}$, then $\mathrm{w}_{3}^{*}(\mathrm{v}, \mathrm{y}, \mathrm{z}) \in S$ and

$$
\left\{w_{3}^{*}(v, y, z) ; u\right\}=w_{4}^{*}(u, v, y, z)
$$

These identities are an extension of the ideas introduced by J. M. Dolan in [4]. They can be verified by expanding $\mathrm{W}_{4}$ along its last column and $\mathrm{W}_{4}^{*}$ along its first column. It is clear from identities (3.3) and (3.4) that $\{u, v, y, z\}$ forms a solution basis for $S\left(S^{*}\right)$ if and only if $\left\{\mathrm{z} ; \mathrm{W}_{3}(\mathrm{u}, \mathrm{v}, \mathrm{y})\right\}=\mathrm{k} \neq 0 \quad\left(\left\{\mathrm{w}_{3}^{*}(\mathrm{v}, \mathrm{y}, \mathrm{z}) ; \mathrm{u}\right\}=\mathrm{k} \neq 0\right)$.

Our first two results establish a connection between two and three dimensional subspaces of $S\left(S^{*}\right)$ and certain second and third order Wronskians. These results are related to the Wronskian identities established by W. J. Kim in $[8]$.

THEOREM 3.1. Let y $\in S\left(S^{*}\right)$ and let $S_{y}^{*}\left(S_{y}\right)$ be the subset of $S^{*}(S)$ defined by 


$$
S_{y}^{*}=\left\{z \in S^{*} \mid\{y ; z\}=0\right\} \quad\left(S_{y}=\{u \in S \mid\{u ; y\}=0)\right.
$$

Then:

(i) The set $S_{y}^{*}\left(S_{y}\right)$ is a three dimensional subspace of $S^{*}(S)$.

(ii) Let $\mathrm{u} \in S\left(S^{*}\right)$. Then $S_{\mathrm{y}}^{*}=S_{\mathrm{u}}^{*}\left(S_{\mathrm{y}}=S_{\mathrm{u}}\right)$ if and only if $\mathrm{y}=\mathrm{ku}$ for some nonzero constant $\mathrm{k}$.

(iii) Let $u, v, z$ be a basis for $S_{y}^{*}\left(S_{y}\right)$. Then $w_{3}^{*}(u, v, z)=$ my $\left(W_{3}(u, v, z)=m y\right)$ for some nonzero constant $m$. In fact, the basis $u, v, z$ may be chosen such that $w_{3}^{*}(u, v, z)=y \quad\left(w_{3}(u, v, z)=y\right)$.

NOTE. The proofs in this section will be given in terms of elements of $S$ only. It will be clear that the same arguments apply equally as well for elements of $S^{*}$.

PROOF. Part (i) follows from the fact that $\{y ; z\}=0$ essentially defines a third order, linear, homogeneous differential equation.

Consider part (ii). Since $\{.,$.$\} is linear in each of its arguments, it is$ easy to see that if $y=k u$, then $S_{y}^{*}=S_{u}^{*}\left(S_{y}=S_{u}\right)$. To show the converse assume that $y, u \in S$ are linearly independent. Since $S_{y}^{*}$ is a three dimensional subspace of $S^{*}$, it follows that if a is any point in $R^{+}$and $i, j$ are any two distinct integers, $0 \leq i, j \leq 3$, then there exists a solution $z \in S_{y}^{*}$ such that $D_{i}^{*} z(a)=D_{j}^{*} z(a)=0$. Fix any $a \in R^{+}$. Suppose $y(a)=0, u(a) \neq 0$. Then it is easy to verify, using the Lagrange identity, that $S_{y}^{*}$ contains the solution $z$ satisfying $\mathrm{D}_{0}^{*} \mathrm{z}(\mathrm{a})=\mathrm{D}_{1}^{*} \mathrm{z}(\mathrm{a})=\mathrm{D}_{2}^{*} \mathrm{z}(\mathrm{a})=0, \mathrm{D}_{3}^{*} \mathrm{z}(\mathrm{a})=1$, and $\mathrm{z} \notin \mathrm{S}_{\mathrm{u}}^{*}$. Thus $S_{\mathrm{y}}^{*} \neq S_{\mathrm{u}}^{*}$. The same argument applies if $u(a)=0, y(a) \neq 0$. Now suppose $y(a) \neq 0$ and $u(a) \neq 0$. From part (i) we can assume that $y(a)=u(a)$. Let $i, 1 \leq i \leq 3$ be the least integer such that $\mathrm{D}_{i} \mathrm{y}(\mathrm{a}) \neq \mathrm{D}_{i} \mathrm{u}(\mathrm{a})$. Such an integer exists since $\mathrm{y}$ and $\mathrm{u}$ are linearly independent. Now choose $z \in S_{y}^{*}$ such that $D_{j}^{*} z(a)=D_{k}^{*} z(a)=0$, $j \neq k, 0 \leq j, k \leq 2$ and $j \neq 3-i, k \neq 3-i$. Then from the Lagrange identify $z \notin S_{u}^{*}$ 
For (iii), let $\{u, v, z\}$ be a basis for $S_{y}^{*}$ and let $w=w_{3}^{*}(u, v, z\}$. Then, from $(3.4),\{\mathrm{W} ; \mathrm{u}\}=\{\mathrm{W} ; \mathrm{v}\}=\{\mathrm{W} ; \mathrm{z}\}=0$, which implies $S_{\mathrm{W}}^{*}=S_{\mathrm{y}}^{*}$, and so $\mathrm{W}=$ my from (ii). The last part of (iii) follows from the fact that $\frac{1}{m} \mathrm{~W}_{3}^{*}(\mathrm{u}, \mathrm{v}, \mathrm{z})=\mathrm{w}_{3}^{*}\left(\frac{1}{\mathrm{~m}} \mathrm{u}, \mathrm{v}, \mathrm{z}\right) \quad\left\{\frac{1}{\mathrm{~m}} \mathrm{~W}_{3}(\mathrm{u}, \mathrm{v}, \mathrm{z})=\mathrm{w}_{3}\left(\frac{1}{\mathrm{~m}}, \mathrm{v}, \mathrm{z}\right)\right\}$.

THEOREM 3.2. Let $y, z \in S\left(S^{*}\right)$ be linearly independent. Then:

(i) $S_{y}^{*} \cap S_{z}^{*}\left(S_{y} \cap S_{z}\right)$ is a two dimensional subspace of $S^{*}(S)$.

(ii) If $\{u, v\}$ is a basis for $S_{y}^{*} \cap S_{z}^{*}\left(S_{y} \cap S_{z}\right)$, then $\mathrm{w}_{2}(y, z)=\mathrm{kW}_{2}^{*}(\mathrm{u}, \mathrm{v}) \quad\left\{\mathrm{w}_{2}^{*}(\mathrm{y}, \mathrm{z})=\mathrm{kW}_{2}(\mathrm{u}, \mathrm{v})\right\}$ for some nonzero constant $\mathrm{k}$. Moreover $u$ and $v$ may be chosen so that $w_{2}(y, z)=w_{2}^{*}(u, v) \quad\left\{w_{2}^{*}(y, z)=w_{2}(u, v)\right\}$. PROOF. Part (i) follows from the fact that the intersection of two distinct three dimensional subspaces of a four dimensional vector space has dimension two.

To prove part (ii), assume that $y, z \in S$ are linearly independent and $\{u, v\}$ is a basis for $S_{y}^{*} \cap S_{z}^{*}$. Then $u$ and $v$ satisfy the "third" order equations

$$
\mathrm{D}_{0} \mathrm{y} \mathrm{D}_{3}^{*} \varphi-\mathrm{D}_{1} \mathrm{y} \mathrm{D}_{2}^{*} \varphi+\mathrm{D}_{2} \mathrm{y} \mathrm{D}_{1}^{*} \varphi-\mathrm{D}_{3} \mathrm{y} \mathrm{D}_{0}^{*} \varphi=0
$$

and

$$
\mathrm{D}_{0} \mathrm{z} \mathrm{D}_{3}^{*} \varphi-\mathrm{D}_{1} \mathrm{z} \mathrm{D}_{2}^{*} \varphi+\mathrm{D}_{2} \mathrm{z} \mathrm{D}_{1}^{*} \varphi-\mathrm{D}_{3} \mathrm{z} \mathrm{D}_{0}^{*} \Psi=0
$$

Multiplying these two equations by $y$ and $z$, respectively, and subtracting, yields the second order equation

$$
\mathrm{W}_{2}(\mathrm{y}, \mathrm{z}) \mathrm{D}_{2}^{*} \varphi-\mathrm{p}\left(\mathrm{yz} "-\mathrm{z} \mathrm{y}^{\prime \prime}\right) \mathrm{D}_{1}^{*} \varphi+\left[\mathrm{y} \mathrm{D}_{3} \mathrm{z}-\mathrm{z} \mathrm{D}_{3} \mathrm{y}\right] \varphi=0
$$

Now $\mathrm{D}_{2}^{*} \varphi=\mathrm{p} \varphi "-\mathrm{q} \varphi$, so that this equation can be written.

$$
\mathrm{W}_{2}(\mathrm{y}, \mathrm{z}) \varphi^{\prime \prime}-\mathrm{W}_{2}^{\prime}(\mathrm{y}, \mathrm{z}) \varphi^{\prime}+\frac{\left[\mathrm{y} \mathrm{D}_{3} \mathrm{z}-\mathrm{z} \mathrm{D}_{3} \mathrm{y}-\mathrm{q}\right] \varphi}{\mathrm{p}}=0
$$

Since $u$ and $v$ satisfy this equation, we have, by Abel's identity,

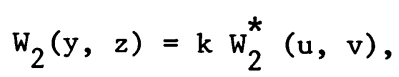

for some nonzero constant $k$, on any interval on which $w_{2}(y, z) \neq 0$. The 
continuity of $\mathrm{w}_{2}, \mathrm{w}_{2}^{*}$ and their derivatives imply that this equation actually holds on $\mathrm{R}^{+}$. Finally, since $\mathrm{kW}_{2}^{*}(\mathrm{u}, \mathrm{v})=\mathrm{W}_{2}^{*}(\mathrm{ku}, \mathrm{v})$, we have (ii) in the case y, $z \in S$.

We conclude this section with a necessary and sufficient condition that each of $(L)$ and $\left(L^{*}\right)$ be nonoscillatory. This condition is stated in terms of the nonoscillation of second and third order Wronskians of linearly independent solutions. In particular, the Wronskian $w_{i}\left(w_{i}^{*}\right), i=2$ or 3 , of linearly independent solutions of $S(S)^{*}$ is nonoscillatory if there is a number $b \in R^{+}$ such that $w_{i}\left(w_{i}^{*}\right)$ is nonzero on $[b, \infty)$; otherwise $w_{i}\left(w_{i}^{*}\right)$ is oscillatory.

THEOREM 3.3. Equations (L) and $\left(L^{*}\right)$ are nonoscillatory if and only if every second and third order Wronskian $\mathrm{w}_{2}, \mathrm{w}_{3}\left\{\mathrm{~W}_{2}^{*}, \mathrm{w}_{3}^{*}\right\}$ of linearly independent solutions of $S\left\{S^{*}\right\}$ is nonoscillatory.

PROOF. Assume that all second and third order Wronskians of linearly independent solutions of (L) are nonoscillatory. Then, in particular, all third order Wronskians $\mathrm{w}_{3}$ are nonoscillatory. By Theorem 3.1 (iii) every solution of $S^{*}$ is the Wronskian of three linearly independent solutions of $S$. Thus we can conclude that $\left(\mathrm{L}^{*}\right)$, and hence $(\mathrm{L})$, are both nonoscillatory.

Now assume that (L) is nonoscillatory. Then Theorem 3.1 (iii) and the fact that $\left(\mathrm{L}^{*}\right)$ is also nonoscillatory implies that all third order Wronskians of linearly independent solutions of (L) are nonoscillatory. Thus, it remains to examine the second order Wronskians. Let y, $z \in S$ be linearly independent and assume $y>0, z>0$ on $[b, \infty)$ for some $b \in R^{+}$. It was shown in [5, Lemma 3.1] that if $u$ is any nonoscillatory solution of (L), then

$$
\prod_{i=0}^{2} D_{i} u \neq 0 \text { on }[c, \infty) \text { for some } c \in R^{+} \text {. }
$$

Therefore, by taking $b$ large enough, we may assume $y>0, z>0, D_{1} y \neq 0$, $D_{i} z \neq 0, i=1,2$, on $[b, \infty)$. Suppose $w_{2}(y, z)$ is oscillatory. Recall the 
subsets $I, D$ and $O$ of $S$. We show first that we cannot have $y \in I, z \in D \cup 0$ (or vice versa). Since $\mathrm{w}_{2}(y, z)=\mathrm{yz}^{\prime}-\mathrm{zy} \mathrm{y}^{\prime}$, it is clear that $y \in I, z \in D$ is impossible. Suppose, therefore that $y \in I$ and $z \in 0$. There are three possible cases for the signs of $\mathrm{z}$ and its "derivatives".

$$
\begin{array}{ll}
\text { (I) } z>0, z^{\prime}>0, D_{2} z<0 & \text { on }[b, \infty), \\
\text { (II) } z>0, z^{\prime}<0, D_{2} z>0, D_{3} z>0 & \text { on }[c, \infty), c \geq b, \\
\text { (III) } z>0, z^{\prime}>0, D_{2} z>0, D_{3} z<0 & \text { on }[c, \infty), c \geq b .
\end{array}
$$

Since $\mathrm{y}>0$ and $\mathrm{y}$ is strongly increasing, we may assume that $\mathrm{D}_{\mathrm{i}} \mathrm{y}>0$, $i=1,2,3$, on $[b, \infty)$. If $z$ satisfies (II), then $w_{2}(y, z)<0$ on $[c, \infty)$. If $z$ satisfies $(I)$, then $W_{2}^{\prime}(y, z)=y z "-z y^{\prime \prime}<0$ on $[b, \infty)$ which implies that $w_{2}$ has constant sign on $[d, \infty)$ for some $d \geq b$. Finally, suppose $z$ satisfies (III). Then it is easy to verify that

$$
\left(\mathrm{pw}_{2}^{\prime}\right)=\left|\begin{array}{cc}
\mathrm{y}^{\prime} & \mathrm{z}^{\prime} \\
\mathrm{D}_{2} \mathrm{y} & \mathrm{D}_{2} \mathrm{z}
\end{array}\right|+\left|\begin{array}{cc}
\mathrm{y} & \mathrm{z} \\
\mathrm{D}_{3} \mathrm{y} & \mathrm{D}_{3} \mathrm{z}
\end{array}\right|,
$$

and as a result of the signs of $y, z$ and their "derivatives", we find that the first term on the right hand side is monotone decreasing and the second term has limit $-\infty$. Thus $\underset{x \rightarrow \infty}{\lim } \mathrm{pW}_{2}^{\prime}=-\infty$. This implies that $\mathrm{w}_{2}^{\prime}<0$ on $[\mathrm{d}, \infty)$ for some $\mathrm{d} \geq \mathrm{b}$ and so $w_{2}$ is eventually of one sign. We can now conclude that if $w_{2}(y, z)$ oscillates, then either $y, z \in I$, or $y, z \in D \cup 0$.

Assume $y, z \in I$ and $w_{2}(y, z)$ oscillates. Choose $u \in D \cup O$ such that $y, z, u$ are linearly independent. Then $W_{3}(y, z, u) \neq 0$ on $[a, \infty)$ for some $a \in R^{+}$, and $\{y, z, u\}$ is a solution basis for the third order differential equation

$$
\left|\begin{array}{cccc}
\varphi & \mathrm{y} & \mathrm{z} & \mathrm{u} \\
\varphi^{\prime} & \mathrm{D}_{1} \mathrm{y} & \mathrm{D}_{1} \mathrm{z} & \mathrm{D}_{1} \mathrm{u} \\
\mathrm{p} \varphi^{\prime \prime} & \mathrm{D}_{2} \mathrm{y} & \mathrm{D}_{2} \mathrm{z} & \mathrm{D}_{2} \mathrm{u} \\
\left(\mathrm{p} \varphi^{\prime \prime}\right)^{\prime} & \mathrm{D}_{3} \mathrm{y} & \mathrm{D}_{3} \mathrm{z} & \mathrm{D}_{3} \mathrm{u}
\end{array}\right|=0
$$


which can be written in the form

$$
w_{3}(y, z, u)\left(p \varphi^{\prime \prime}\right)^{\prime}-w_{3}^{\prime}(y, z, u)\left(p \varphi^{\prime \prime}\right)+f(x) \varphi^{\prime}+g(x) \varphi=0 .
$$

Now, the fact that $u$ and $w_{2}(y, u)$ are nonoscillatory implies that there exists $\mathrm{b} \geq \mathrm{a}$ such that $\mathrm{u} \neq 0$ and $\mathrm{w}_{2}(\mathrm{y}, \mathrm{u}) \neq 0$ on $[\mathrm{b}, \infty)$. Therefore, according to Ahmad [2, p292] (see also, G. Polya $\lceil 12\rceil$ ), (8) is disconjugate on $\lceil b, \infty$ ). This implies that the adjoint of equation (3.5) is disconjugate on $\lceil\mathrm{b}, \infty)$

(see J. H. Barrett [3]). But $\psi(x)=w_{2}(y, z) / w_{3}(y, z, u)$ is an oscillatory solution of the adjoint equation, and we have a contradiction. The same method of proof can be used if we assume that $y, z \in D \cup O$ and $\mathrm{w}_{2}(y, z)$ oscillates.

\section{SUBSPACES OF S AND S:}

In this section we consider the structure of the three dimensional subspaces of $S$ and $S^{*}$, and as a corollary, we also identify certain two dimensional subspaces. We will be making use of the subsets $I, \mathcal{D}$ and 0 of $S$ and $I^{*}, D^{*}$ and $O^{*}$ of $S^{*}$ defined in Section 2 , and we shall also be concerned with the "complimentary" subspaces $S_{y}^{*}\left(S_{y}\right)$ determined by the solutions y of $S\left(S^{*}\right)$. In this regard, recall that if $y \in S\left(S^{*}\right)$, then $S_{y}^{*}\left(S_{y}\right)$ is the three dimensional space of solutions of the third order equation

$$
\begin{aligned}
& \mathrm{D}_{0} \mathrm{y} \mathrm{D}_{3}^{*} \varphi-\mathrm{D}_{1} \mathrm{y} \mathrm{D}_{2}^{*} \varphi+\mathrm{D}_{2} \mathrm{y} \mathrm{D}_{1}^{*} \varphi-\mathrm{D}_{3} \mathrm{y} \mathrm{D}_{0}^{*} \varphi=0, \\
& \left.\mathrm{D}_{0}^{*} \mathrm{y} \mathrm{D}_{3} \Psi-\mathrm{D}_{1}^{*} \mathrm{y} \mathrm{D}_{2} \Psi+\mathrm{D}_{2}^{*} \mathrm{y}_{1} \Psi-\mathrm{D}_{3}^{*} \mathrm{y} \mathrm{D}_{0} \Psi=0\right\}
\end{aligned}
$$

In [6], M. Hanan defined two classes of third order linear differential equations. In particular, a third order equation is in class $\mathrm{C}_{I}$ on $[a, \infty)$ if any solution with double zero at some point $b>a$ is nonzero on $[a, b)$, and it is in class $C_{I I}$ on $[a, \infty)$ if any solution with a double zero at $b \geq a$, is nonzero on $(b, \infty)$.

THEOREM 4.1. Let $\mathrm{y} \in S$. 
(i) If $y \in I$, then $S_{y}^{*}=[z, u, v]$ (= the space spanned by $z, u, v$ ), where $z \in I^{*}, u, v \in 0^{*}, w_{2}^{*}(u, v) \neq 0$ on $[a, \infty)$ for some $a \geq 0$, and the third order equation (9) is in class $C_{I I}$ on $\left.\Gamma a, \infty\right)$.

(ii) If $y \in D$, then $S_{y}^{*}=[w, u, v]$, where $w \in D^{*}, u, v \in 0^{*}, w_{2}^{*}(u, v) \neq 0$ on $[a, \infty)$ for some $a \geq 0$, and the third order equation (4.1) is in class $\mathrm{C}_{\mathrm{I}}$ on $[0, \infty)$.

(iii) If $y \in 0$, then $S_{y}^{*}=[z, w, u]$, where $z \in I^{*}, w \in D^{*}$ and $u \in 0^{*}$ The corresponding statements hold if $y \in S^{*}$.

PROOF. As in Section 3, we will prove the theorem only for the case $y \in S$.

(i). Suppose $y \in I$, and assume, without loss of generality, that $y$ is eventually positive. Then there exists $a \geq 0$ such that $D_{1} y>0,1=0,1,2,3$, on $[a, \infty)$. Choose any $b \geq a$ and let $z$ be a solution of (9), i.e. let $z$ be an element of $S_{y}^{*}$ such that $z$ has a double zero at $b$. Then it is easy to verify from (9) that $\mathrm{D}_{2}^{*} \mathrm{z}(\mathrm{b})$ and $\mathrm{D}_{3}^{*} \mathrm{z}(\mathrm{b})$ cannot have opposite sign. We may assume, therefore, that $\mathrm{D}_{2}^{*} \mathrm{z}(\mathrm{b}) \geq 0, \mathrm{D}_{3}^{*} \mathrm{z}(\mathrm{b}) \geq 0$ with at least one inequality being strict, and so, by Theorem 2.1, $z \in I^{*}$ and equation (4.1) is in class $C_{I I}$ on $[a, \infty)$. Now choose $w \in D$ and consider the two dimensional subspace $S_{y}{ }^{*} n S_{w}^{*}$. If $\mathrm{u} \in S_{\mathrm{y}}^{*} \cap S_{\mathrm{w}}^{*}$, then $\{\mathrm{y} ; \mathrm{u}\}=\{\mathrm{w} ; \mathrm{u}\}=0$ from which it follows that $\mathrm{u} \in 0^{*}$. Thus $S_{y}^{*} \cap S_{w .}^{*}=[u, v]$, where $u, v \in O^{*}$. Finally, from Theorem 3.2 (ii), there is a nonzero constant $\mathrm{k}$ such that $\mathrm{w}_{2}{ }^{*}(\mathrm{u}, \mathrm{v})=\mathrm{k} \mathrm{w}_{2}(\mathrm{y}, \mathrm{w}) \neq 0$ on $[\mathrm{a}, \infty)$. Therefore $S_{y}^{*}=[z, u, v]$ and the proof of part (i) is complete.

(ii). Suppose $y \in D$, and assume, without loss of generality, that $(-1)^{i} D_{i} y>0$ on $[0, \infty)$. Choose any $b>0$ and let $z$ be a solution of (4.1) such that $z$ has a double zero at $b$. Then it is easy to see from (4.1) that $D_{2}^{*} z(b)$ and $D_{3}^{*} z(b)$ must have opposite sign, say $D_{2}^{*} z(b)>0, D_{3}^{*} z(b)<0$. Thus, by Theorem 2.1, we have $(-1){ }^{i} D_{i}^{*} z>0, i=0,1,2,3$, on $[0, b)$ and equation $(4.1)$ is in class $C_{I}$ on $[0, \infty)$. Now, for each positive integer $n$, let $z_{n}$ be a solution 
of (4.1) which satisfies $z_{n}(n)=D_{1}^{*} z_{n}(n)=0, D_{2}^{*} z_{n}(n)>0, D_{3}^{*} z_{n}(n)<0$. Then by a standard "sequence argument" (see A. C. Lazer [9, Theorem 1.17 or Ahmad [17), we can construct a solution w of (4.1) such that $\operatorname{sgn} w=\operatorname{sgn} D_{2}^{*} w \neq \operatorname{sgn} D_{1}^{*} w=\operatorname{sgn} D_{3}^{*}$ on $[0, \infty)$, i.e. such that $w \in D^{*}$. Now choose any $y \in I$ and assume $D_{i} y>0, i=0,1,2,3$ on $[a, \infty), a \geq 0$. Then by using the same argument as in part (i), we can conclude that $S_{y}^{*}=[w, u, v]$, where $w \in 0^{*}, u, v \in 0^{*}$ and $\mathrm{w}_{2}^{*}(\mathrm{u}, \mathrm{v}) \neq 0$ on $[\mathrm{a}, \infty)$.

(iii). Suppose $y \in 0$. Choose any $z \in I$ and any $w \in D$. Then $S_{y}^{*} \cap S_{z}^{*} \cap S_{w}^{*}$ is a one dimensional subspace of $S^{*}$, and if $u \in S_{y}^{*} \cap S_{z}^{*} \cap S_{w}^{*}$, then u must be in $0^{*}$ because $\{z ; u\}=\{w ; u\}=0$. Thus $S_{y}^{*}$ contains an element $u$ in $0^{*}$. Now, suppose that $y$ is oscillatory, and let $\left\{z_{1}, z_{2}, z_{3}\right\}$ be a basis for $s_{y}^{*}$. Then, by Theorem 3.1 (iii), $\mathrm{w}_{3}^{*}\left(\mathrm{z}_{1}, \mathrm{z}_{2}, \mathrm{z}_{3}\right)=$ my for some nonzero constant $\mathrm{m}$. Let $\mathrm{b}$ be $\mathrm{a}$ zero of $y$. Then $w_{3}^{*}\left(z_{1}, z_{2}, z_{3}\right)(b)=0$ which implies that there exists a nontrivial linear combination $z$ of the solutions $z_{1}, z_{2}, z_{3}$ such that $z$ has a triple zero at $\mathrm{b}$. We may assume that $\mathrm{D}_{3}^{*} \mathrm{z}(\mathrm{b})>0$ and conclude, by Theorem 2.1, that $D_{i}^{*} z>0, i=0,1,2,3$, on $(b, \infty)$ so that $z \in I^{*}$. Let $\left\{b_{n}\right\}$ be the sequence of zeros of $y$. Then for each positive integer $n$ there is a solution $z_{n}$ of (4.1) such that $D_{i}^{*} z_{n}\left(b_{n}\right)=0, i=0,1,2$ and $D_{3}^{*} z_{n}\left(b_{n}\right)<0$. Thus, by Theorem 2.1, $(-1)^{i} D_{i}^{*} z_{n}>0, i=0,1,2,3$ on $\left[0, b_{n}\right)$, and so, by using the "sequence argument" cited above we can construct a solution $w$ in $S_{y}^{*}$ such that $w \in D^{*}$. Finally, it is easy to verify that $S_{y}^{*}=[z, w, u]$.

Now suppose that $\mathrm{y}$ is nonoscillatory. As observed in the proof of Theorem 3.3, y and its derivatives must satisfy one of the following three sets of inequalities on an interval $[a, \infty)$.

$$
\begin{aligned}
& \text { (I) } \mathrm{y}>0, \mathrm{y}^{\prime}>0, \mathrm{D}_{2} \mathrm{y}<0 \\
& \text { (II) } \mathrm{y}>0, \mathrm{y}^{\prime}<0, \mathrm{D}_{2} \mathrm{y}>0, \mathrm{D}_{3} \mathrm{y}>0 \\
& \text { (III) } \mathrm{y}>0, \mathrm{y}^{\prime}>0, \mathrm{D}^{2} \mathrm{y}>0, \mathrm{D}_{3} \mathrm{y}<0
\end{aligned}
$$


In each case $y$ has two consecutive derivatives $D_{i} y, D_{i+1} y, 0 \leq i \leq 2$ which have the same sign on $[a, \infty)$, and two consecutive derivatives $D_{j} y, D_{j+1} y, 0 \leq j \leq 2$ which have opposite sign on $[a, \infty)$. Choose any $b \geq a$. Let $z$ be a solution of (4.1) such that $\mathrm{D}_{\mathrm{m}}^{*} \mathrm{z}(\mathrm{b})=0$ for the two $\mathrm{m}^{\prime} \mathrm{s}$ with the property that $\mathrm{D}_{\mathrm{m}}^{*} \mathrm{z}(\mathrm{b})$ is not a coefficient of either $D_{i} y(b)$ or $D_{i+1} y(b)$. It then follows that the remaining two "derivatives" of $z$ at $b$ cannot have opposite sign. Assuming that at least one of these "derivatives" is positive, we have $z \in I^{*}$. Now let $\left\{b_{n}\right\}$ be an increasing sequence in $[a, \infty)$ with $\lim _{n \rightarrow \infty} b_{n}=\infty$. For each positive integer $n$, let $z_{n}$ be a solution of (4.1) such that $D_{m}^{*} z_{n}\left(b_{n}\right)=0$ for the two m's with the property that $D_{m}^{*} z_{n}\left(b_{n}\right)$ is not a coefficient of either $D_{j} y\left(b_{n}\right)$ or $D_{j+1} y\left(b_{n}\right)$. Then we can conclude from equation (9) that the remaining two "derivatives" of $z_{n}$ at $b_{n}$ must have opposite sign. Thus the consecutive "derivatives" of $z_{n}$ must have opposite sign on $[0, n)$, and the "sequence argument" allows us to construct a solution $w$ of (4.1) such that $w \in D^{*}$. Finally, it is easy to verify that the three solutions, $u, z$, and $w$ are linearly independent, and this completes the proof of the theorem.

There are a variety of consequences of Theorem 4.1 which describe the structure of the two and three dimensional subspaces of $S$ and $S^{*}$. We list these results in the following corollaries.

COROLIARY: (1) Every three dimensional subspace of $S\left(S^{*}\right)$ has a nonoscillatory solution.

(2) Equation (L) $\left\{\left(L^{*}\right)\right\}$ is oscillatory if and only if every three dimensional subspace of $S\left(S^{*}\right)$ has an oscillatory solution.

(3) Every three dimensional subspace of $S\left(S^{*}\right)$ contains a pair of solutions whose Wronskian is nonzero on $[a, \infty)$ for some $a \geq 0$.

PROOF. Let $T$ be any three dimensional subspace of $S\left(S^{*}\right)$, and let $\left\{y_{1}, y_{2}, y_{3}\right\}$ be a basis for $T$. Then $y=w\left(y_{1}, y_{2}, y_{3}\right) \quad\left\{y=w^{*}\left(y_{1}, y_{2}, y_{3}\right)\right\}$ is 
an element of $S^{*}(S)$, and $T=S_{y}\left(T=S_{y}^{*}\right)$.

We now consider the two dimensional subspaces of $S$ and $S^{*}$. We note that corresponding to any two dimensional subspace $T$ of $S$ there is a unique two dimensional subspace $T^{*}=S_{y}^{*} \cap S_{z}^{*}$ of $S^{*}$, where $\{y, z\}$ is any basis for $T$. Similar1y, a two dimensional subspace $T^{*}$ of $S^{*}$ determines a unique two dimensional subspace $T$ of $S$.

COROLLARY 2. Let $T$ be a two dimensional subspace of $S$. Then $T$ satisfies exactly one of the following:

(1) $T=[y, u], y \in I, u \in O$, and $T^{*}=[z, v\urcorner, z \in I^{*}, v \in O^{*}$

(2) $T=[y, w], y \in I, w \in D$, and $T^{*}=[u, v], u \in 0^{*}, v \in 0^{*}$ and every combination of $\mathrm{u}$ and $\mathrm{v}$ is in $0^{*}$.

(3) $T=[\mathrm{w}, \mathrm{u}], \mathrm{w} \in \mathcal{D}, \mathrm{u} \in \mathcal{O}$, and $T^{*}=[\mathrm{z}, \mathrm{v}], \mathrm{z} \in \mathrm{D}^{*}, \mathrm{v} \in 0^{*}$

(4) $T=[u, v], u \in 0^{*}, v \in 0^{*}$ and every combination of $u$ and $v$ is in $0^{*}$, and $T^{*}=[y, w], y \in I^{*}, w \in D^{*}$.

Clearly, the corresponding statements hold if $T^{*}$ is a two dimensional subspace of $S^{*}$.

PROOF. The four cases follow from the fact that if $T$ is any two dimensional subspace of $S$, then $T \cap(O \cup D) \neq \emptyset$ and $T \cap(O \cup I) \neq \emptyset$. This fact can be established by considering the intersections $T \cap S_{y}$ and $T \cap S_{w}$, where $y \in I^{*}$, $\mathrm{w} \in D^{*}$, and using Theorem 4.1 .

The structure of $T$ specified in cases (1) - (3) is easy to verify. The structure specified in (4) follows from the fact that if $y \in 0^{*}$, then any two dimensional subspace of $S_{y}$ must contain either an element of $I$, or an element of D. Thus $T^{*}$ cannot contain an element of $0^{*}$ from which it follows that $T^{*}=[y, w\rceil, y \in I^{*}, w \in 0^{*}$.

We conclude by noting that if the equations $(L)$ and $\left(L^{*}\right)$ are oscillatory, then the structure of a two dimensional subspace can be determined by 
looking at the Wronskian of a basis for the subspace. Recall that if (L) and $\left(L^{*}\right)$ are oscillatory, then every solution in 0 and $O^{*}$ is oscillatory.

COROLLARY 3. Assume that equation (L), and hence $\left(\mathrm{L}^{*}\right)$, are oscillatory. Let $T$ be a two dimensional subspace of $S$, let $\left\{y_{1}, y_{2}\right\}$ be a solution basis for $T$, and let $W(x)=W_{2}\left(y_{1}, y_{2}\right)$.

(1) If $\mathrm{W}$ is oscillatory, then either $T=\lceil\mathrm{y}, \mathrm{u}], \mathrm{y} \in I, \mathrm{u} \in \mathcal{O}$ and $T^{*}=[z, v], z \in I^{*}, v \in 0^{*}$, or $T=[w, u], w \in D, u \in 0$ and $T^{*}=[y, v]$, $y \in 0^{*}, v \in 0^{*}$.

(2) If $\mathrm{W}$ is nonoscillatory, then either $T=\lceil\mathrm{y}, \mathrm{w}\rceil, \mathrm{y} \in \mathrm{O}, \mathrm{w} \in \mathrm{D}$ and $T^{*}=[u, v], u, v \in 0^{*}$, every linear combination of $u$ and $v$ is in $0^{*}$, and the zeros of two independent solutions separate on $[a, \infty)$ for some $a \geq 0$, or vice versa, i.e. $T=[\mathrm{u}, \mathrm{v}], \mathrm{u}, \mathrm{v} \in \mathrm{0}$, etc., and $T^{*}=[\mathrm{y}, \mathrm{w}], \mathrm{y} \in I^{*}, \mathrm{w} \in 0^{*}$.

\section{REFERENCES}

1. Ahmad, S., "On the oscillation of solutions of a class of fourth order differential equations", Pacific J. Math., 34 (1970), 289-299.

2. Ahmad, S., "Oscillation properties of third order linear differential equations and their adjoints", Journal of Math. and Phys. Sciences, VIII (1974), 291-298.

3. Barrett, J. H., "Oscillation theory of ordinary linear differential equations", Advances in Mathematics, $\underline{3}$, Academic Press, New York, (1969), 415-509.

4. Dolan, J. M., "On the relationship between the oscillatory behavior of a linear third order differential equation and its adjoint", J. Differential Equations, $\underline{7}$ (1970, 367-388.

5. Etgen, G. J., and W. E. Taylor, Jr., "On the oscillation of a class of fourth order differential equations", Rocky Mtn J. Math., $\underline{6}$ (1976), 71-84.

6. Hanan, M., "Oscillation criteria for third-order linear differential equations", Pacific J. Math., 11 (1961), 919-944.

7. Keener, M. S., "On solutions of certain self-adjoint differential equations of fourth order", J. Math. Anal. and App1., 33 (1971), 278-304. 
8. Kim, W. J., "A relation satisfied by solutions of the adjoint equation", Kyungpook Mathematical J., 13 (1973), 127-131.

9. Lazer, A. C., "The behavior of solutions of the differential equation $y^{\prime \prime \prime}+p(x) y^{\prime}+q(x) y=0 "$, Pacific J. Math., 17 (1966), 435-466.

10. Leithton, W. and $\mathrm{Z}$. Nehari, "On the oscillation of solutions of self-adjoint linear differential equations of the fourth order", Trans. Am. Math. Soc., 89 (1958), 325-377.

11. Polya, G., "On the mean-value theorem corresponding to a given linear homogeneous differential equation", Trans. Am. Math. Soc., 24 (1922), 312-324.

12. Pudei, V., o vlastnostech reseni diferencialni rovnice $y^{(4)}+p(t) y^{\prime \prime}+$ $q(t) y=0$, Casopis pro Pestorani Matematiky, 93 (1968), 201-216

13. Pudei, V., Uber die Eigenschaften der Losungen linearer Defferentia1 Gleicheingen gerader Ordnung", Casopis pro Pestovani Matematiky, 94 (1969), 401-425. 


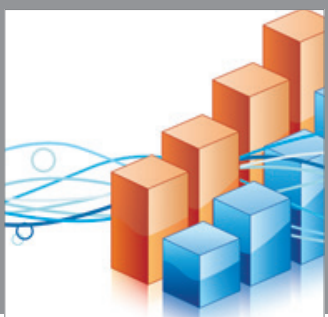

Advances in

Operations Research

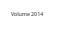

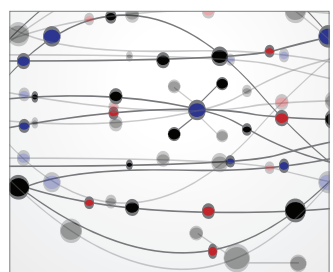

\section{The Scientific} World Journal
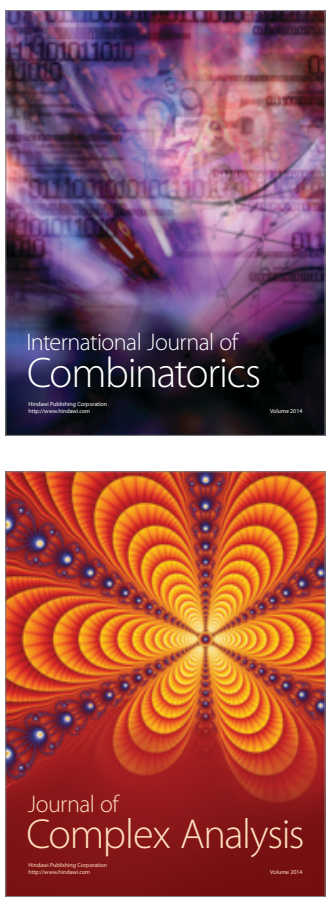

International Journal of

Mathematics and

Mathematical

Sciences
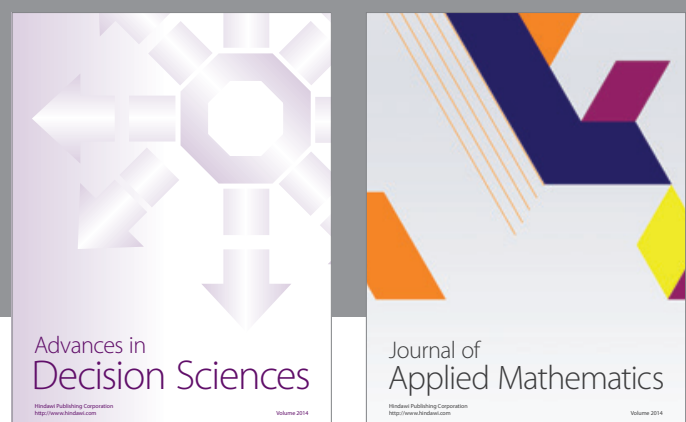

Journal of

Applied Mathematics
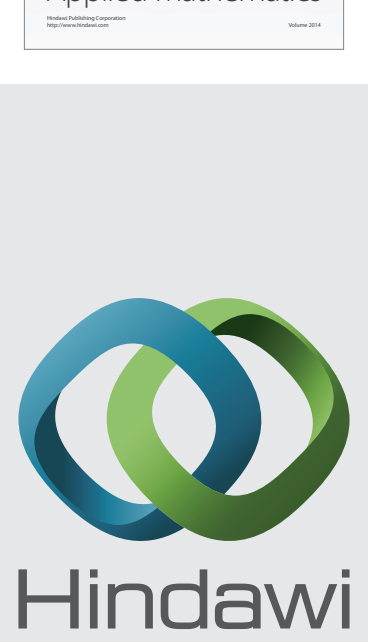

Submit your manuscripts at http://www.hindawi.com
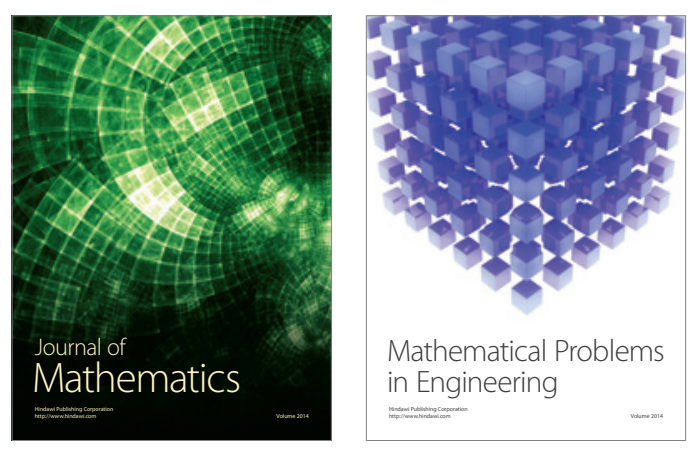

Mathematical Problems in Engineering
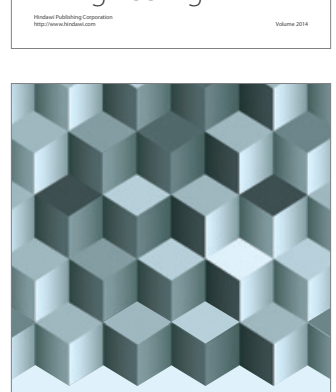

Journal of

Function Spaces
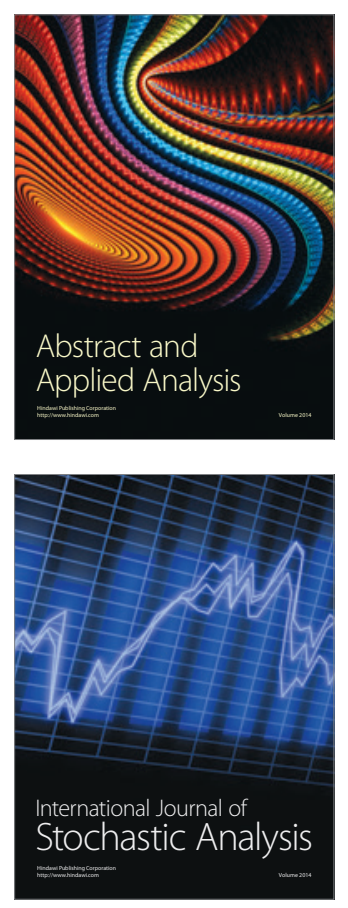

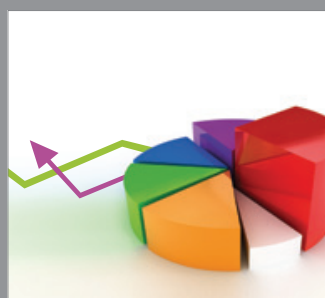

ournal of

Probability and Statistics

Promensencen
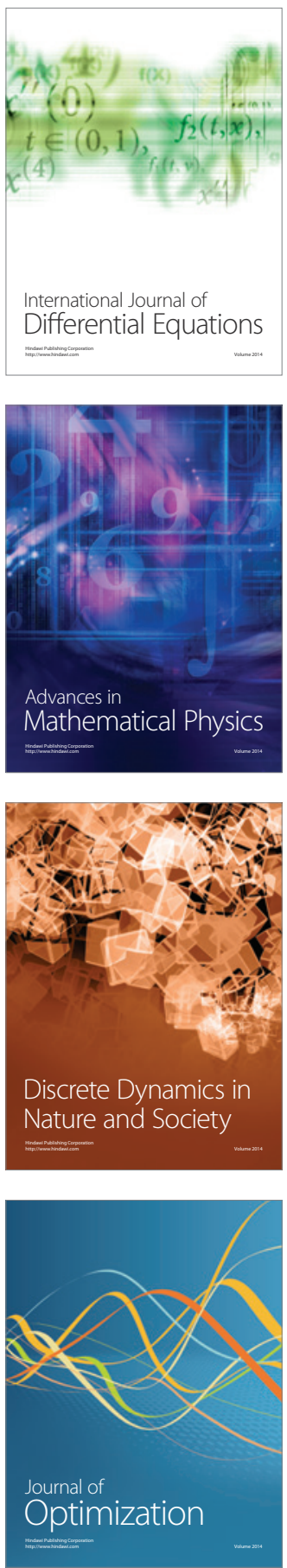